\title{
First aid management under the guidance of new cardiopulmonary resuscitation guidelines
}

\author{
Lu Wang, Aqian Wang, Yu Han, Xiaxin Dong \\ School of Medical Sciences, Yan'an University, Yan'an 716000, Shaanxi Province, P.R. China
}

Keywords: 2015, cardiopulmonary resuscitation, guidelines, new changes, analysis

Abstract: At present, cardiopulmonary resuscitation is the basic technology for rescuing patients, and it plays an important role in emergency work. In 2015, the American Heart Association released a new cardiopulmonary resuscitation guide. This article examines the new changes in the 2015 version of the Cardiopulmonary Resuscitation Guide based on the 2010 edition of the Cardiopulmonary Resuscitation Guide, and looks forward to helping medical staff to scientifically and quickly rescue patients.

\section{Introduction}

Cardiac arrest will threaten the patient's life, and CPR will be needed to restore the patient's heartbeat and breathing. As early as 1950, American professors studied cardiopulmonary resuscitation. In 1980, cardiopulmonary resuscitation gradually matured, and several cardiopulmonary resuscitation methods, such as mouth-to-mouth breathing, chest compression, and external motor defibrillation, were identified.

\section{Cardiopulmonary resuscitation guide changes in the life chain}

In 2015, the American Heart Association issued a new cardiopulmonary resuscitation guide, which pointed out that the life chain could divide patients with cardiac arrest into patients in hospitals and outside hospitals to determine the treatment of patients. Regardless of where the patient has a cardiac arrest problem, these patients need to be treated in the hospital. Usually, the hospital's intensive care unit will provide treatment for patients with cardiac arrest. The community needs to treat patients with cardiac arrest outside the hospital. The community can use social media technologies (such as mobile phones) to help patients search for nearby people with real-time cardiopulmonary resuscitation and are willing to help. In the 2015 edition of the Cardiopulmonary Resuscitation Guide, the key link in the adult survival chain outside the hospital is the same as the 2010 version of the cardiopulmonary resuscitation guidelines, which emphasizes the adult basic life support process [1]. Non-medical personnel need to know the patient's condition as soon as possible, and give patients first aid, using cardiopulmonary resuscitation, rapid defibrillation and other treatments to restore the heartbeat and breathing of patients with cardiac arrest. In order to avoid the patient's sudden cardiac arrest, the hospital uses a special monitoring system to monitor the actual situation of the patient. If a patient has a cardiac arrest problem, the health care provider will perform a cardiopulmonary resuscitation in a team: early warning system, rapid response team, and 
emergency medical team system, which are treated by professional medical staff.

\section{Changes in Basic Life Support Technologies}

The chest compression position, compression depth, compression time, and number of artificial ventilations will all affect the quality of cardiopulmonary resuscitation.

\subsection{Compression Frequency}

The 2010 cardiopulmonary resuscitation guidelines indicate that the frequency of chest compressions is more than 100 times a minute. In the 2015 edition of the Cardiopulmonary Resuscitation Guidelines, the frequency of chest compressions in adults and children was 100 to 120 beats per minute. When giving patients cardiac resuscitation, the number of chest compressions is important for the recovery of autonomic circulation and recovery of neurological function after survival. Studies have shown that the number of chest compressions will affect the survival rate of patients. If the chest compression frequency is too fast, it will affect the patient's thoracic rebound and compression depth [2]. The study found that when the frequency of chest compressions was 125 beats/min, the patient's spontaneous circulation recovery and survival rate were higher. If the frequency of chest compressions increased, the patient's spontaneous circulation recovery and survival rate would decrease. In the chest compression of patients with cardiac arrest, not only to ensure that the compression frequency is reasonable, but also to ensure the persistence of compression, to ensure the success rate of cardiopulmonary resuscitation, improve the survival rate of patients with cardiac arrest. Thus, the 2015 edition of the Cardiopulmonary Resuscitation Guidelines clarifies the importance of chest compressions in cardiopulmonary resuscitation.

\subsection{Chest Compression Depth}

The 2010 edition of the Cardiopulmonary Resuscitation Guide states that when performing extra-thoracic compression on an adult patient, the compression depth is required to be above $5 \mathrm{~cm}$, When the chest is pressed against the baby, the depth of compression is $4 \mathrm{~cm}$, when the chest is pressed against the child, the depth of compression is $5 \mathrm{~cm}$. The 2015 edition of the Cardiopulmonary Resuscitation Guide states that when performing chest compressions on adult patients, it is necessary to ensure a compression depth of between 5 and $6 \mathrm{~cm}$, when performing chest compressions on infants, it is necessary to ensure a compression depth of $4 \mathrm{~cm}$, When performing chest compression on children, it is necessary to ensure a compression depth of $5 \mathrm{~cm}$. and adolescents who are entering adolescence should ensure that the compression depth is the same as that of an adult. Through chest compressions, the patient's thoracic pressure is increased or the patient's heart is compressed to supply blood to important organs to ensure the basic requirements for patient survival. The study found that after non-medical staff performed cardiopulmonary resuscitation in patients with cardiac arrest, the depth of compression of the survivors was about 5.36 centimeters, and the depth of compression of the deceased was about 4.84 centimeters. This shows that the depth of chest compression has an important impact on the survival of patients with cardiac arrest. Although medical personnel often emphasize the need to press the patient's chest hard, it is difficult for ordinary people to determine whether the compression depth is appropriate. Therefore, non-medical personnel generally have a problem of excessively shallow compression when performing chest compressions on patients with cardiac arrest. Therefore, in the 2015 edition of the cardiopulmonary resuscitation guidelines, it is pointed out that when the chest compression is performed on adults, the compression depth should be 5 to $6 \mathrm{~cm}$. The main reason is that the compression depth is too deep, which will cause complications, including: sternal and rib fractures, 
Pneumothorax, hemothorax, cardiopulmonary injury, etc.

\subsection{Thoracic Rebound}

In the 2010 edition of the Cardiopulmonary Resuscitation Guide, after each press is over, the rescuer is required to perform a next press after the patient's thoracic rebound to ensure that the patient's heart is filled before the next press. This requirement applies to both adults and children, but in the 2015 edition of the Cardiopulmonary Resuscitation Guide, the rescuer is required to ensure that it is at a distance from the patient's chest in the compression gap to ensure that the patient's thorax rebounds sufficiently after each compression. (The full thoracic rebound means that during the cardiopulmonary resuscitation, the patient's sternum will return to its original position). Negative pressure will be generated during the patient's thoracic backfilling, prompting venous return and cardiopulmonary reflow [3]. If the rescuer still relies on the patient's chest during the compression gap, it will affect the rebound of the patient's thorax. If the patient's thorax does not rebound enough, it will increase the patient's thoracic pressure, affecting the patient's venous return, coronary perfusion pressure, etc., which will adversely affect the patient's survival rate.

\subsection{Keep the Persistence of Compression}

In the 2010 edition of the cardiopulmonary resuscitation guidelines, rescuers need to ensure the continuity of chest compressions as much as possible, reduce the number and time of interruption of chest compressions, and ensure the rationality of the number of chest compressions. In the 2015 edition of the Cardiopulmonary Resuscitation Guide, rescuers need to ensure the continuity of chest compressions as much as possible. In the first-aid treatment of patients with cardiac arrest outside the hospital, the persistence of chest compression should be ensured. The main reason is that chest compression is important for the patient to resume spontaneous circulation and improve the survival rate of patients. Therefore, for patients with cardiac arrest who do not have advanced airway for cardiopulmonary resuscitation, the proportion of chest compressions in cardiopulmonary resuscitation should be increased as much as possible. When performing chest compression on the patient, it is necessary to ensure the continuity of the compression as much as possible to increase the patient's venous return, coronary perfusion pressure, etc., and improve the survival rate of the patient.

\subsection{Ventillation Strategy}

For patients with cardiac arrest who can be shocked outside the hospital, the rescuer can use three 200 cycles of continuous chest compressions, and the patient is given auxiliary airway equipment to delay pressure ventilation. When the chest compressions are not stopped or interrupted, positive pressure ventilation will also cause the patient's heartbeat to stop and the patient's thoracic venous return will decrease, resulting in a decrease in the patient's cardio-cerebral perfusion. When using single-person cardiopulmonary resuscitation, the patient is required to perform two artificial respirations after each 30 compressions, and each artificial respiration time is guaranteed to be more than 1 second. In the 2010 edition of the Cardiopulmonary Resuscitation Guide, when using double cardiopulmonary resuscitation, construct an advanced passage, and ensure that the patient is given an artificial respiration every 6 to 8 seconds. At this time, it is necessary to note that the rescuers do not need to ensure that breathing is synchronized with chest compressions. To ensure that medical staff can easily learn, remember, and implement cardiopulmonary resuscitation. In the 2015 edition of the Cardiopulmonary Resuscitation Guide, it is proposed to perform an artificial respiration every six seconds, and to ensure that artificial 
respiration is synchronized with chest compressions.

\subsection{First Electric Shock Or Cardiopulmonary Resuscitation}

The 2010 edition of the Cardiopulmonary Resuscitation Guide states that when the rescuer finds an adult cardiac arrest and an automated external defibrillator is available in the field, the rescuer needs to perform a chest compression on the patient and use an automatic external defibrillator to treat the patient as soon as possible. If the hospital or other institution has an automatic external defibrillator, the medical staff needs to perform cardiopulmonary resuscitation and use an automatic external defibrillator as soon as possible. Its main purpose is to restore the patient's heartbeat and breathing as soon as possible, and defibrillation as soon as possible. If the medical staff does not witness the patient's cardiac arrest, an automatic external defibrillator or electrocardiogram should be used to check the patient's heart rhythm during cardiopulmonary resuscitation, and defibrillation should be started according to the patient's actual condition [4]. When there are a large number of rescuers on site, the patient should be cardiopulmonary resuscitation while taking the automatic external defibrillator. In the 2015 edition of the Cardiopulmonary Resuscitation Guide, if an external external defibrillator is available on site, chest compressions should be performed during adult cardiac arrest and defibrillation should be performed as soon as possible using an automated external defibrillator. If no one is witnessing the cardiac arrest of an adult patient, or if there is no automatic external defibrillator on site, cardiopulmonary resuscitation is required when other rescuers take an automatic external defibrillator. At the same time, the rescuer needs to defibrillate as soon as possible according to the actual situation of the patient. In addition, studies have shown that patients with cardiac arrest due to ventricular fibrillation or pulseless ventricular tachycardia, the defibrillation process will make an important factor affecting the patient's survival rate, as the time before defibrillation increases, the patient's chance of death will continue to increase Therefore, the rescuer needs to defibrillate the patient as soon as possible to ensure the survival rate of the patient.

\subsection{The Importance of Teamwork}

The 2010 version of the Cardiopulmonary Resuscitation Guidelines stipulates that the basic life support steps need to be performed in a traditional order so that the rescuer can master the sequence of cardiopulmonary resuscitation. In the 2015 edition of the Cardiopulmonary Resuscitation Guide, not only the rescue order of the single rescuer but also the importance of team recovery is emphasized. Rescuers who have participated in relevant training need to perform respiratory and pulse examinations on patients with cardiac arrest. Thereby achieving the goal of shortening the time of the first chest compression. The use of an emergency response system ensures the smooth operation of the treatment and the flexibility of subsequent processing. Basic life support professional training can not only teach the trainee's personal cardiopulmonary resuscitation technology, but also help the trainee understand and master the advanced life support process, so that it can be used as a member of the recovery team to improve the survival rate of patients with cardiac arrest.

\section{Advanced Life Support Management Study}

\subsection{Airway Management}

According to the Cardiopulmonary Resuscitation Guidelines issued by the American Heart Association in 2010, it is necessary to continuously monitor the concentration of carbon dioxide in 
the patient's breath during cardiac arrest. The medical staff needs to determine the position of the catheter based on the patient's end-tidal carbon dioxide concentration, monitor the quality of the cardiopulmonary resuscitation, and detect whether the patient has recovered heartbeat and breathing. In the cardiopulmonary resuscitation guidelines released in 2015, it is indicated that for intubated patients, after 20 minutes of cardiopulmonary resuscitation, If the patient's end-tidal carbon dioxide concentration is less than $10 \mathrm{mmHg}$ detected by the carbon dioxide waveform, it is difficult for the patient to recover the heartbeat and breathing, and the survival probability is low [5]. Although medical staff cannot determine treatment options based solely on this indicator, medical staff can consider various factors to determine when to stop cardiopulmonary resuscitation.

\subsection{Cardiopulmonary Resuscitation Drug}

Studies have shown that administration of adrenaline and vasopressin to patients with cardiac arrest can help patients return to spontaneous circulation. In the 2010 edition of the Cardiopulmonary Resuscitation Guide, in the patient's cardiac arrest, the medical staff can inject 40 units of vasopressin into the patient's vein/bone to help the patient recover spontaneously. In the 2015 edition of the Cardiopulmonary Resuscitation Guide, it was stated that in the patient's cardiac arrest, the medical staff's combined use of adrenaline and vasopressin was not good enough, so medical staff could only use a drug called adrenaline. The study found that the earlier the patient was treated with adrenaline, the higher the survival rate of the patient when the patient had a cardiac arrest problem and was not treated with electric shock. At this stage, no relevant studies have shown that patients using lidocaine after cardiac arrest can effectively improve the survival rate of patients. A current study has shown that patients with lidocaine are treated with ventricular fibrillation/pulmonary ventricular tachycardia due to cardiac arrest, and patients with ventricular fibrillation/pulmonary ventricular tachycardia after resuming spontaneous circulation The probability of rapid recurrence is reduced, but no studies have shown whether there is an adverse reaction in long-term use. Thus, when a patient has a cardiac arrest, the medical staff can be treated with lidocaine.

\subsection{Temperature Management}

In the 2010 edition of the Cardiopulmonary Resuscitation Guidelines, the body temperature of an adult patient who is required to return to the autonomic circulation after a cardiac fibrillation cardiac arrest outside the hospital is controlled at 32 to $34^{\circ} \mathrm{C}$, and this state is maintained for 12 to 24 hours. For patients with cardiac arrest in the hospital, after the patient resumed autonomic circulation, compared with the recovery of patients with controlled body temperature and those who did not control body temperature, the neurological prognosis of patients with controlled body temperature was found to be good [6]. Studies have shown that patients with cardiac arrest after the recovery of autonomic circulation, the patient's body temperature is controlled within a reasonable range, the patient recovery is better. In short, controlling the body temperature of patients with cardiac arrest at 32 to $36^{\circ} \mathrm{C}$, and maintaining more than 24 hours, plays an important role in the rehabilitation of patients.

\subsection{Hemodynamic Parameters After Cardiopulmonary Resuscitation}

The study found that patients with cardiac arrest had a systolic blood pressure below $90 \mathrm{mmHg}$, or when the mean arterial pressure was below $65 \mathrm{mmHg}$, the patient's mortality would increase. When the systolic blood pressure of patients with cardiac arrest is above $100 \mathrm{mmHg}$, the patient's recovery is better. In the treatment of patients with cardiac arrest, medical staff need to scientifically 
adjust the patient's blood pressure to ensure that the patient's blood pressure is within a reasonable range to enhance the patient's survival rate.

\section{Conclusion}

In short, with the development of the medical industry, cardiopulmonary resuscitation has also developed rapidly, and the guidelines for cardiopulmonary resuscitation are constantly being updated, which is of great significance for the rescue of patients with cardiac arrest. Medical staffs need to master the relevant ability of cardiopulmonary resuscitation, continuously improve the recovery process, improve the medical staff's ability to treat, and play an important role in improving the success rate of cardiopulmonary resuscitation.

\section{Acknowledgement}

Technical training and promotion of cardiopulmonary resuscitation in Yan'an City(2017HM-08) Policy-support and Education Project of Yan'an University yazzzd18-152

\section{References}

[1] Hongliang Ren, Hongjie Xi. Interpretation of the 2015 American Heart Association Guidelines for Cardiac Arrest and Resuscitation during Pregnancy [J]. International Journal of Obstetrics and Gynecology, 2017, 44(3): $347-349$.

[2] Bincheng Lou, Jihong Zhu. 2015 American Heart Association (AHA) Cardiopulmonary Resuscitation and Cardiovascular Emergency Update Guide Interpretation of Advanced Cardiopulmonary Resuscitation [J]. Clinical Misdiagnosis and Mistreatment, 2016(2): 71-74.

[3] Hui Zhang, Yunfang Li, Shanshan Yao. Application of practicing teaching method while watching video in the training of cardiopulmonary resuscitation guide for 2015 internship nursing students[J]. Journal of Chinese Nursing, 2016, 23(16): 12-14.

[4] Li Yan. The value of the 2015 cardiopulmonary resuscitation guide and the 2010 guideline [J]. Heilongjiang Medicine, 2016, 29(1): 140-142.

[5] Jianxu Wei, Mengxia Zhu, Yuyu Yang, Wei Huang. A comparative study of cardiopulmonary resuscitation and mechanical cardiopulmonary resuscitation for cardiopulmonary resuscitation [J]. Health Road, 2017, 16 (05): 36.

[6] Chulin Chen, Li Gui, Ting Qi, Xian Wang, Shuang Li, Chen Qiu. Research progress and application status of cardiopulmonary resuscitation reminder/feedback device [J].Jiangsu Journal of Nursing,2016,33(20):28-31+ 47.

Wang Lu, MS, professor, Who was born in chunhua county, shaanxi province in 1972, mainly engaged in anatomy teaching in Medical College of Yan'an University 